\title{
Communication
}

\section{Detection of Two Phenomena Opposite to the Expected Ones}

\author{
Boris I. Kochelaev
}

Physics Institute, Kazan Federal University, 42008 Kazan, Russia; bkochelaev@gmail.com

Received: 1 September 2020; Accepted: 19 September 2020; Published: 24 September 2020

check for updates

\begin{abstract}
Both phenomena mentioned in the title were revealed by the electron paramagnetic resonance (EPR) method. The first phenomenon was found in superconducting La metal with Er impurities - the spin relaxation rate of the erbium impurities was sharply decreasing after transition into the superconducting state instead of the expected, i.e., the well-known Hebel-Slichter peak. The second unexpected phenomenon was discovered in the $\mathrm{YbRh}_{2} \mathrm{Si}_{2}$ compound-an excellent EPR signal from the $\mathrm{Yb}$ ions was observed at temperatures below the Kondo temperature determined thermodynamically, while according to the existing belief the EPR signal should not be observed at these temperatures due to the Kondo effect. In this tribute to K. Alex Müller, I describe the nature of the detected phenomena.
\end{abstract}

Keywords: superconductivity; Kondo effect; spin relaxation rate; magnetic resonance

\section{Introduction}

It is reasonable to remind readers that the electron paramagnetic resonance (EPR) study by Alex Müller on transition metal ions in the $\mathrm{SrTiO}_{3}$ compound having the perovskite structure was the first step on the way to the discovery of high-temperature superconductivity (HTSC) [1]. His similar EPR study of different properties including the relation to their superconductivity in other perovskites gave him an impulse to search for superconductivity with a higher critical temperature, see [2]. The result is well known (1986). It is remarkable that Alex in his talk in 1969 at the International Conference dedicated to the 25th year of the EPR discovery by E. K. Zavoisky at Kazan University described advantages of the EPR method to study phase transitions, especially for their second type. For me, his opinion was not new due to our meetings before at the AMPERE colloquiums.

Magnetic impurities in transition metals and intermetallic compounds were used a rather long time ago as EPR probes to study the interactions and properties of these materials. It is useful for the following discussion to give some necessary results of these investigations (details can be found in the review by Barnes [3]).

In the case of a low concentration of impurities, their spin relaxation rate is defined mainly by interactions with conduction electrons and phonons. The exchange coupling of impurities with conduction electrons in the case of the axial symmetry can be presented by the following Hamiltonian:

$$
H_{e x}=-\sum_{i}\left\{J_{\perp}\left[S_{i}^{x} \sigma^{x}\left(\mathbf{r}_{i}\right)+S_{i}^{y} \sigma^{y}\left(\mathbf{r}_{i}\right)\right]+J_{\|} S_{i}^{z} \sigma^{z}\left(\mathbf{r}_{i}\right)\right\}
$$

where $J_{\perp}, J_{\|}$are the exchange integrals, $S_{i}^{x, y, z}$ are the spin components of the impurity, and $\sigma^{x, y, z}\left(\mathbf{r}_{i}\right)$ are the spin density components of the conduction electrons at the $\mathbf{r}_{i}$ position. The spin-lattice relaxation rate of conduction electrons $\Gamma_{\sigma L}$ is defined usually by their scattering on different defects of the lattice and on phonons due to their spin-orbital and spin-phonon interactions. If this relaxation is effective enough, the spin temperature of conduction electrons remains in the equilibrium state with other 
degrees of freedom. The EPR line width is defined in this case mainly by the relaxation rate $\Gamma_{S S}$ (the so-called Korringa relaxation rate) due to the exchange interaction (Equation (1)). The spin-lattice relaxation rate of impurities due to other interactions $\Gamma_{S L}$ in metals can be usually neglected. In the simplest case where $J_{\perp}=J_{\|}=J$, the Korringa relaxation rate is given by the following equation:

$$
\Gamma_{S S}=\frac{4 \pi}{\hbar}\left(\rho_{F} J\right)^{2} k_{B} T
$$

where $\rho_{F}$ is the density of electronic orbital states at the Fermi energy level, $k_{B}$ is the Boltzmann constant, and $T$ is the temperature. The vice versa spin relaxation rate of conduction electrons to the equilibrium state of impurities (the Overhauser relaxation rate) $\Gamma_{\sigma \sigma}$ and $\Gamma_{S S}$ satisfy the following detailed balance equation:

$$
\frac{\Gamma_{\sigma \sigma}}{\Gamma_{S S}}=\frac{\chi_{S}^{0} g_{\sigma \sigma}^{2}}{\chi_{\sigma}^{0} g_{S}^{2}} ; \chi_{S}^{0}=N S(S+1) \frac{\left(g_{S} \mu_{B}\right)^{2}}{3 k_{B} T}, \chi_{\sigma}^{0}=\rho_{F} \frac{\left(g_{\sigma} \mu_{B}\right)^{2}}{2}
$$

where $\chi_{S}^{0}, \chi_{\sigma}^{0}$ are the spin susceptibilities of non-interacting impurities and the conduction electrons, $g_{S}, g_{\sigma}$ are the corresponding $g$-factors, and $N$ and $S$ are the concentration and spin of impurities, respectively. However, a very different situation appears if the spin systems of impurities and conduction electrons are strongly coupled and their Zeeman frequencies $\hbar \omega_{S}=g_{S} \mu_{B} H_{0}, \hbar \omega_{\sigma}=g_{\sigma} \mu_{B} H_{0}$ are very close $\left(H_{0}\right.$ is the external magnetic field). This case can be represented by the following relations:

$$
\Gamma_{S S}, \Gamma_{\sigma \sigma} \gg \Gamma_{S L}, \Gamma_{\sigma L},\left|\omega_{S}-\omega_{\sigma}\right|
$$

In this situation, both spin systems cannot be considered in the equilibrium states. The motion equations of magnetic impurities and conduction electrons are coupled by the additional coefficients $\Gamma_{S \sigma}$ and $\Gamma_{\sigma S}$, which coincide in the isotropic case with $\Gamma_{\sigma \sigma}$ and $\Gamma_{S S}$ correspondingly. The spin relaxation of the whole system is realized then by the two steps as follows: first, the spin systems of magnetic impurities and conduction electrons achieve the common spin temperature and, second, they both relax to the equilibrium state of the lattice (the electron bottleneck regime). The spin dynamics of both spin systems in the bottleneck regime is also sufficiently changed since the collective spin excitations of impurities and conduction electrons appear. We are interested in the EPR signal of the mode, corresponding to the spin oscillations of impurities and conduction electrons in the same phase. In the simplest case $\omega_{S}=\omega_{\sigma}\left(g_{S}=g_{\sigma}\right)$ under the relaxation-dominated bottleneck regime, the spin relaxation rate of this mode $\Gamma_{\text {coll }}$ can be presented as follows (neglecting $\Gamma_{S L}$ ):

$$
\Gamma_{\mathrm{coll}}=\Gamma_{S \sigma} B, B=\Gamma_{\sigma L} /\left(\Gamma_{\sigma S}+\Gamma_{\sigma L}\right)
$$

where $B$ is the bottleneck factor-the less this factor, the stronger the bottleneck regime; in the case $B=1$, the bottleneck is absent. If the impurities' concentration is large enough $\left(\chi_{S}^{0} \gg \chi_{\sigma}^{0}\right)$, then $\Gamma_{\text {coll }}$ is strongly reduced and proportional to temperature:

$$
\Gamma_{\mathrm{coll}} \simeq \frac{\Gamma_{S \sigma}}{\Gamma_{\sigma S}} \Gamma_{\sigma L}=\frac{\chi_{\sigma}^{0}}{\chi_{S}^{0}} \Gamma_{\sigma L}=b T, b=\frac{3 k_{B} \rho_{F} \Gamma_{\sigma L}}{2 N S(S+1)}
$$

Since the spin-lattice relaxation of conduction electrons $\Gamma_{\sigma L}$ does not depend on temperature, the behavior according to (Equation (6)) imitates the Korringa relaxation rate (Equation (2)), despite the bottleneck situation. Nevertheless, it should be mentioned that in the strongly anisotropic case $\left(J_{\perp} \neq J_{\|}\right)$ it was found that the EPR line width narrowing is rather weak despite the bottleneck regime [4]. In the 
case of a parallel orientation of the external magnetic field to the symmetry axis for the spin relaxation rate of the collective mode (instead of Equation (6)), roughly the following result was obtained:

$$
\Gamma_{\text {coll }} \sim \frac{\chi_{\sigma}^{0}}{\chi_{S}^{0}} \Gamma_{\sigma L}+\frac{\left(J_{\perp}-J_{\|}\right)^{2}\left(g_{\perp}^{2}+g_{\sigma}^{2}\right)}{2 J_{\perp} J_{\|} g_{\perp} g_{\sigma}} \Gamma_{S \sigma}
$$

If distances between impurities become relatively short, one should also take into account their magnetic dipole-dipole interactions and the Ruderman-Kittel-Kasuya-Yosida (RKKY) spin-spin interactions via conduction electrons. The latter indirect interaction between two impurities with spins $\mathbf{S}_{1}$ and $\mathbf{S}_{2}$ at the distance $R$ between them is as follows:

$$
\begin{aligned}
& H_{\text {ind }}(R)=J_{\text {ind }}\left(\mathbf{S}_{1} \mathbf{S}_{2}\right) f\left(2 k_{F} R\right) ; \\
& J_{\text {ind }}=\frac{9 \pi Z^{2} J^{2}}{2 E_{F}}, f(x)=\frac{\cos x}{x^{3}}-\frac{\sin x}{x^{4}} .
\end{aligned}
$$

where $k_{F}$ is the electron wave vector at the Fermi surface, $Z$ is the number of conduction electrons per lattice atom, and $E_{F}$ is the Fermi energy. Although this interaction is rather long range, it should be limited by the free path distance $l_{p}$ of the conduction electrons: $f(x) \rightarrow f(x) \exp \left(-R / l_{p}\right)$. The dipole-dipole interactions give an additional broadening of the EPR signal from impurities, and the RKKY interaction leads to its narrowing of the Anderson-Weiss type. This contribution to the EPR line width is independent of temperature being responsible for the residual line width (at $T=0)$.

\section{Peculiarities of the EPR Signal from Impurities in Superconductors}

It is well known that NMR gives very important information about properties of superconductors confirming the Bardeen-Cooper-Schrieffer (BCS) theory [5,6]. It was found that the Hebel-Slichter peak appears due to a sharp increase of the nuclear spin relaxation rate to the superconducting electrons via their hyperfine interactions $H_{I \sigma}$ :

$$
H_{I \sigma}=A \sum_{i}\left\{I_{i}^{x} \sigma^{x}\left(\mathbf{r}_{i}\right)+I_{i}^{y} \sigma^{y}\left(\mathbf{r}_{i}\right)+I_{i}^{z} \sigma^{z}\left(\mathbf{r}_{i}\right)\right\}
$$

According to the BCS theory, the observed jump of the Korringa-type nuclear spin relaxation rate happens as a consequence of the sharp increase of the electron density states near the edge of the superconducting energy gap and due to the coherence factor of the Cooper pairing. A further lowering of temperature leads to an exponential decrease of the Korringa relaxation rate due to the thermal depopulation of the electronic state above the mentioned gap. The BCS theory explains the NMR result for the nuclear spin relaxation rate rather well (see Figure 1). In the case of superconductors of the second type, an additional broadening of the NMR signal due to the inhomogeneous penetration of the external magnetic field appears.

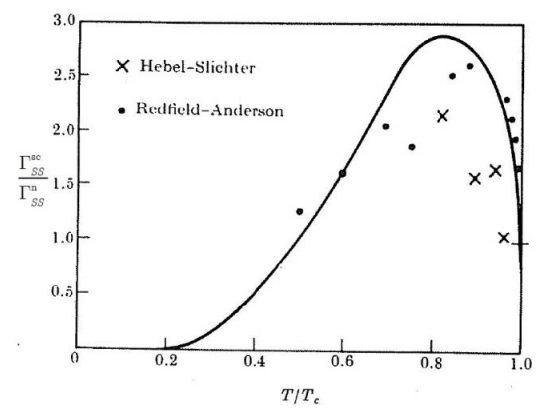

Figure 1. The temperature dependence of the nuclear spin relaxation rates' ratio in the superconducting and normal states of aluminum. The crosses and dots are results obtained in [6,7] accordingly. The solid line was obtained according to the Bardeen-Cooper-Schrieffer (BCS) theory in [8] (after [5], Section 3.3). 
Since the structure of the hyperfine interactions (Equation (9)) is identical to the exchange interactions of impurities (Equation (1)), it was quite natural to expect similar behavior of the Korringa relaxation rate of the localized electrons. The only thing to do, for the interpretation of the EPR signal from magnetic impurities, was to substitute the hyperfine parameter $A$ for the exchange integral $J$. The first observations of the EPR signal from impurities in superconductors were similar to the NMR results $[9,10]$. It is clearly seen in Figure 2.

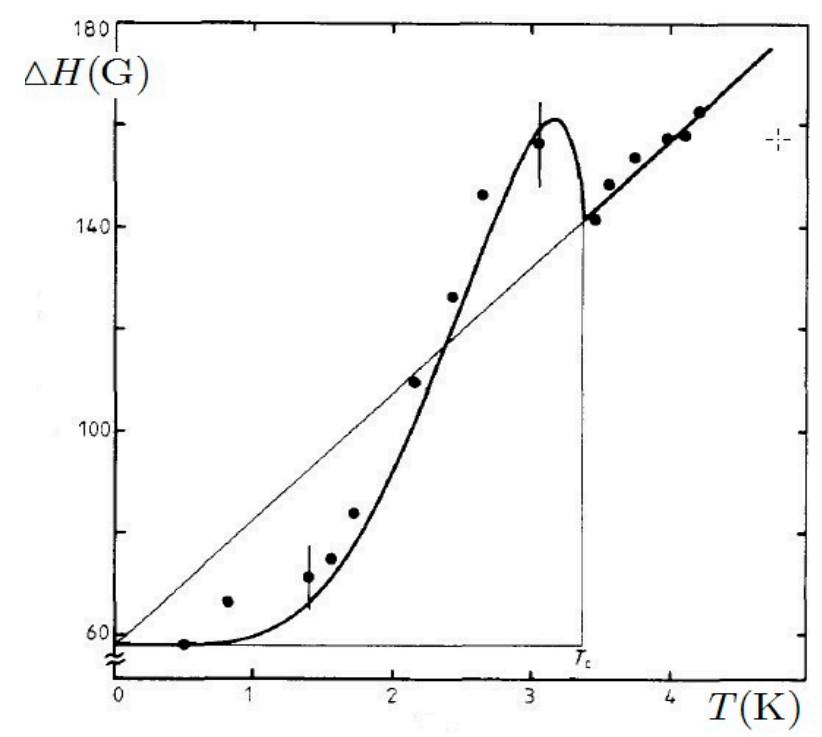

Figure 2. The temperature dependence of the electron paramagnetic resonance (EPR) line width for the $\mathrm{Gd}^{3+}$ in the $\mathrm{LaRu}_{2}$ compound. The dots are the experimental results from [9,10], the solid line was calculated in [11] (after [11]).

However, an opposite phenomenon was rather soon observed by the EPR on the $\mathrm{Er}^{3+}$ ions in lanthanum ( $\beta$ - phase) - the EPR line width, instead of an expected increase after transition into the superconducting state, was sharply narrowed [12]. Later, a more detailed investigation of such a behavior of the EPR signal was performed for the same system [13]. Experimental results of the EPR line width dependence as functions of the temperature for different concentration of the Er ions are shown in Figure 3.

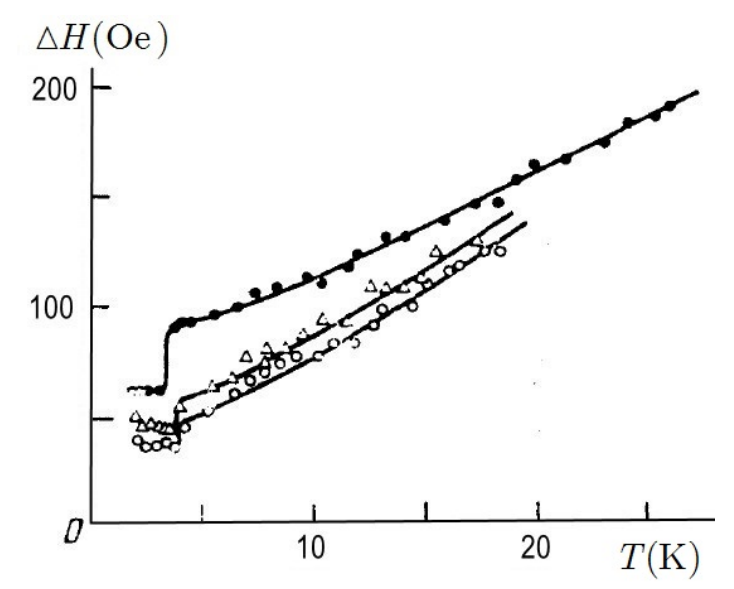

Figure 3. Temperature dependence of the EPR line width for $\mathrm{Er}^{3+}$ ions in the superconducting Lanthanum ( $\beta$-phase) with $\mathrm{Er}^{3+}$ ions: black dots-2 at.\% Er, triangles-1 at.\% Er, open circles- 0.5 at. $\%$ Er (after [13]). 
In order to understand this phenomenon, it was proposed that the superconducting transition leads to a realization of the inequalities (Equation (4)) due to the increase of the spin relaxation rate of impurities to the conduction electrons [14]. As a result, the spin relaxation process of the total spin system transforms into the strong bottleneck regime with narrowing of the EPR signal. The qualitative explanation of this effect can be given in the following way. The effective spin relaxation rate in superconductors can be represented by the equation similar to Equation (5), with $B^{\text {sc }}$ as the superconducting bottleneck parameter:

$$
\Gamma_{\mathrm{coll}}^{\mathrm{sc}} \simeq \Gamma_{S \sigma}^{\mathrm{sc}} B^{\mathrm{sc}}, B^{\mathrm{sc}}=\Gamma_{\sigma L}^{\mathrm{sc}} /\left(\Gamma_{\sigma S}^{\mathrm{sc}}+\Gamma_{\sigma L}^{\mathrm{sc}}\right)
$$

In the case of $\Gamma_{\sigma S}^{\mathrm{sc}} \ll \Gamma_{\sigma L}^{\mathrm{sc}}$, the bottleneck regime is absent. The EPR line width in this situation is defined by the Korringa relaxation rate with the Hebel-Slichter peak similar to the NMR results. In the opposite case of $\Gamma_{\sigma S}^{\mathrm{sc}} \gg \Gamma_{\sigma L}^{\mathrm{sc}}$, we have the strong bottleneck regime and the effective spin relaxation rate is proportional now to the spin-lattice relaxation rate of the superconducting electrons, as follows:

$$
\Gamma_{\mathrm{coll}}^{\mathrm{sc}} \simeq\left(\Gamma_{S \sigma}^{\mathrm{sc}} / \Gamma_{\sigma S}^{\mathrm{sc}}\right) \Gamma_{\sigma L}^{\mathrm{sc}}=\left(\chi_{\sigma}^{\mathrm{sc}} / \chi_{S}^{0}\right) \Gamma_{\sigma L}^{\mathrm{sc}}
$$

It was found earlier that the spin relaxation rate of superconducting electrons $\Gamma_{\sigma L}^{\mathrm{sc}}$ becomes temperature dependent and decreases by lowering the temperature [15]. The exchange integral of the localized and the conduction electrons contained in the relaxation rates of the fraction (Equation (11)) is mutually canceled. The spin susceptibility of the superconducting electrons $\chi_{\sigma}^{\mathrm{sc}}$ now also depends on the temperature getting much lower due to the Cooper pairing. These qualitative arguments were realized using the Feynman diagram technique, and it was shown that the Hebel-Slichter peak is absent in the case of the strong bottleneck regime [16].

The detailed temperature dependence of the effective spin relaxation rate of magnetic impurities in superconductors was investigated in [11] for different values of the bottleneck parameters $B^{\mathrm{n}}$ in the normal state of superconductors. The result is shown in Figure 4 for the normalized effective spin relaxation rate $R_{\text {coll }}^{\mathrm{sc}}=\Gamma_{\mathrm{coll}}^{\mathrm{sc}} / \Gamma_{\mathrm{coll}}^{\mathrm{n}}$, with $\Gamma_{\text {coll }}^{\mathrm{n}}$ as the effective spin relaxation rate in the normal state and for the particular set of the object parameters.

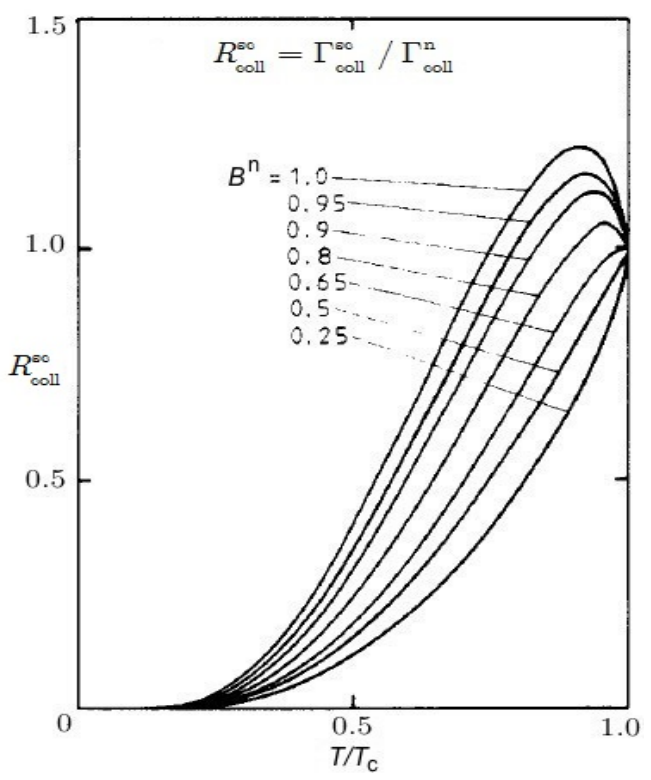

Figure 4. The normalized effective spin relaxation rate $R_{\mathrm{coll}}^{\mathrm{sc}}$ as a function of the reduced temperature $T / T_{\mathrm{C}}$ for the several values of the normal state bottleneck factor $B^{\mathrm{n}}$ (after [11]). 
The important role of the bottleneck regime in the normal state for the temperature dependence of the spin relaxation rate and, therefore, for the EPR signal from impurities in the superconducting state is now clearly seen. It must be mentioned that, in the case of nuclear spins, the bottleneck regime cannot appear because of a great difference in the resonance frequencies of the NMR and the EPR and very small nuclear spin susceptibility.

Another important contribution to the narrowing process of the EPR signal from impurities can arise from the transformation of the RKKY interaction due to the superconducting transition. In the superconducting state, in addition to the usual expression (Equation (8)), a non-oscillating long-range term $H_{\text {ind }}^{\text {sc }}$ of antiferromagnetic character appears [17]. Since the corresponding expression is rather cumbersome, we mention only that this term slowly varies with distance and has the range of the order of the superconducting coherence length $\xi$, roughly $f^{s c}(R) \sim(1 / R) \exp (-R / \xi)$. The typical value of the coherence length is rather large, i.e., $\xi \gg a_{0}, a_{0}$ is the lattice spacing. The detailed theory of the RKKY narrowing of the EPR signal in superconductors was developed in [18]. This type of the EPR line width narrowing has to be taken into account especially in the case of a weak bottleneck regime when interpreting the EPR results in superconductors.

\section{The EPR Signal below the Kondo Temperature}

The Kondo effect appears in metals with magnetic impurities in the case of their antiferromagnetic exchange interactions with conduction electrons $(J<0)$. To consider scattering of conduction electrons by the impurity at the $\mathbf{r}_{i}=0$ site, we use the secondary quantization for the conduction electrons in (Equation (1)):

$$
H_{\mathrm{ex}}^{0}=-\frac{1}{2} \sum_{\mathbf{k}, \mathbf{k}^{\prime}}\left[J_{\|} S_{z}\left(c_{\mathbf{k} \uparrow}^{+} c_{\mathbf{k}^{\prime} \uparrow}-c_{\mathbf{k} \downarrow}^{+} c_{\mathbf{k}^{\prime} \downarrow}\right)+J_{\perp} S_{-}\left(c_{\mathbf{k} \uparrow}^{+} c_{\mathbf{k}^{\prime} \downarrow}+S_{+} c_{\mathbf{k} \downarrow}^{+} c_{\mathbf{k}^{\prime} \uparrow}\right)\right]
$$

where $S_{ \pm}=S_{x} \pm i S_{y}$ and $c_{\mathbf{k} \downarrow}^{+}, c_{\mathbf{k}^{\prime} \uparrow}$ are operators of creation and annihilation of electrons with the corresponding wave vectors and spin orientations. The probability of the electron transition from the state $\left|\mathbf{k}_{1} \uparrow\right\rangle$ into the state $\left|\mathbf{k}_{2} \downarrow\right\rangle$ in first-order perturbation theory is defined by the matrix element $\left\langle\mathbf{k}_{2} \downarrow\left|H_{\mathbf{e x}}^{0}\right| \mathbf{k}_{1} \uparrow\right\rangle=-J_{\perp} S_{+} / 2$. To obtain the second order for this matrix element, it is convenient to use the perturbation theory in the operator form [19]. We consider the energy band symmetric relative to the Fermi level, the electron energy $\xi_{\mathbf{k}}$ measured from the latter in the range $(-W, W)$. To simplify calculations, it is reasonable to use the following case: $k_{B} T \ll W$ and $S=1 / 2$, see [20]. Taking into account the second-order contribution $\delta H_{\mathrm{ex}}^{0}$, one can obtain the following expression for the isotropic symmetry:

$$
\left\langle\mathbf{k}_{2} \downarrow\left|H_{\mathbf{e x}}^{0}+\delta H_{\mathbf{e x}}^{0}\right| \mathbf{k}_{1} \uparrow\right\rangle \approx-\left(J S_{+} / 2\right)\left\{1-\rho_{F} J \ln \left[W /\left(k_{B} T\right)\right]\right\}
$$

It is clear that in the case of $J<0$ the matrix element increases with the lowering of temperature. The second term starts to exceed the first one approximately at the following temperature:

$$
T_{\mathrm{K}} \sim\left(W / k_{B}\right) \exp \left[-1 /\left(\rho_{\mathrm{F}}|J|\right)\right]
$$

which bears the name of Kondo, who obtained these results in order to explain the temperature dependence of the resistivity [21]. The Kondo effect also becomes apparent in the temperature dependences of the specific heat, magnetic susceptibility, and the EPR signal from magnetic impurities (see [22]).

One can see that at the temperature $T \rightarrow 0$ the matrix element goes to infinity. It means that the second order of the perturbation theory is not enough. For isotropic symmetry, it has been shown that taking into account all orders of the logarithmic terms leads to very important consequences, i.e., below the Kondo temperature the magnetic moments of impurities are screened by the cloud of the conduction electrons and the EPR line width is of the $T_{K}$ order (see $\left.[23,24]\right)$. These arguments created a 
widely accepted opinion that below the Kondo temperature the EPR signal cannot be observed on the localized electrons in metals.

It was a great surprise for many experts to observe the EPR signal from the $\mathrm{Yb}^{3+}$ ions in the $\mathrm{YbRh}_{2} \mathrm{Si}_{2}$ compound at the temperature $T=1.6 \mathrm{~K}$ and even below, whereas its Kondo temperature is $T_{\mathrm{K}}=25 \mathrm{~K}[25,26]$, see Figure 5. It was also especially unexpected since this compound is a Kondo lattice system with heavy fermions.

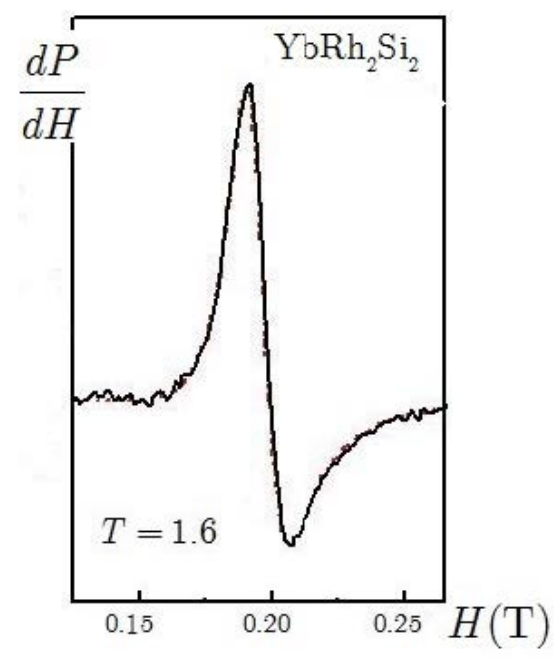

Figure 5. The EPR signal obtained in $\mathrm{YbRh}_{2} \mathrm{Si}_{2}$ below the Kondo temperature $\left(T_{\mathrm{K}}=25 \mathrm{~K}\right)$ (after [26]).

An explanation of this phenomenon can be given on the basis of similar reasons leading to the disappearance of the Hebel-Slichter peak in superconductors, described in the previous Section 2. Since the matrix element (Equation (13)) strongly increases below the Kondo temperature, the same should happen with the Korringa relaxation rate $\Gamma_{S S}$. As a result, the bottleneck regime and the collective spin excitation of the $\mathrm{Yb}^{3+}$ ions with the conduction electrons should appear. Then, the effective spin relaxation rate can be described by Equation (6), where the exchange integral $J$ is absent, that leads to a sufficient reducing of $\Gamma_{\text {eff }}$ and the corresponding narrowing of the EPR line width.

However, doubts about this scenario can appear due to the extremely anisotropic $g$-factors of the $\mathrm{Yb}^{3+}$ ions $\left(g_{\perp}=3.56, g_{\|}=0.17\right)$. It is related to the lowest Kramers doublet as a consequence of the tetragonal symmetry of the crystal electric field. One can expect that exchange integrals $J_{\perp}$ and $J_{\|}$have similar anisotropy and that, for the effective spin relaxation rate, one should use Equation (7) instead of Equation (6). It can lead to an increase of the EPR line width instead of its narrowing. In order to understand the real situation, it was necessary to take into account all the orders of the perturbation theory in calculations of the effective spin relaxation rate for the anisotropic case.

To solve this task, it was convenient to use the method known as "Poor Man's Scaling" developed by Anderson [27]. The main idea of this method was to incorporate the total contribution from the high energy levels into the renormalized parameters of the starting Hamiltonian, in our anisotropic case $J_{\perp} \rightarrow J_{\perp}^{\mathrm{R}}$ and $J_{\|} \rightarrow J_{\|}^{\mathrm{R}}$. The second-order contribution for the initial anisotropic parameters is the following:

$$
\delta J_{\|}=\rho_{F} J_{\perp}^{2} \ln \left(\frac{W}{k_{B} T}\right), J_{\perp} \delta J_{\perp}=J_{\|} \delta J_{\|}
$$

According to Anderson's idea, we divide the energy band into the low and high energy levels:

$$
0<\left|\xi_{\mathbf{k}}\right|<\widetilde{W}, \widetilde{W}<\left|\xi_{\mathbf{k}}\right|<W
$$


For the renormalized contributions, we can obtain the following equations, representing the scaling law:

$$
\begin{aligned}
& \delta \widetilde{J}_{\|}=\rho_{F} \widetilde{J}_{\perp}^{2}\left[\ln \left(\frac{W}{k_{B} T}\right)-\ln \left(\frac{\widetilde{W}}{k_{B} T}\right)\right]=\rho_{F} \widetilde{J}_{\perp}^{2} \ln \left(\frac{W}{\widetilde{W}}\right), \\
& \widetilde{J}_{\perp} \delta \widetilde{J}_{\perp}=\widetilde{J}_{\|} \delta \widetilde{J}_{\|} .
\end{aligned}
$$

These equations are valid in the range $\left(k_{B} T \leq \widetilde{W} \leq W\right)$ for the running band value $\widetilde{W}$. An integration of the second equation gives the following:

$$
\widetilde{J}_{\perp}^{2}-\widetilde{J}_{\|}^{2}=\text { Const }=J_{\perp}^{2}-J_{\|}^{2}
$$

The last equality can be accepted since this equation is valid for any values of $\widetilde{W}$ in the range mentioned above, including $\widetilde{W}=W$. To solve the first Equation (17), we consider it for the case of a small difference $W-\widetilde{W}=\delta \widetilde{W}$. Introducing the dimensionless values $U_{\perp}=\rho_{F} J_{\perp}$ and $U_{\|}=\rho_{F} J_{\|}$, we obtain from Equations (17) and (18) the following equation:

$$
\begin{aligned}
& \delta \widetilde{U}_{\|}=\widetilde{U}_{\perp}^{2} \ln \left(\frac{\widetilde{W}+\delta \widetilde{W}}{\widetilde{W}}\right)=\widetilde{U}_{\perp}^{2} \frac{\delta \widetilde{W}}{\widetilde{W}}=\left(\widetilde{U}_{\|}^{2}+U_{0}^{2}\right) \frac{\delta \widetilde{\widetilde{W}}}{\widetilde{W}}, \\
& \widetilde{U}_{\perp}^{2}-\widetilde{U}_{\|}^{2}=U_{0}^{2}=\rho_{F}\left(J_{\perp}^{2}-J_{\|}^{2}\right) .
\end{aligned}
$$

The integration of this differential equation was performed in the following ranges of the running values: $U_{\|} \leq \widetilde{U}_{\|} \leq U_{\|}^{\mathrm{R}}$ and $k_{B} T \leq \widetilde{W} \leq W$, where $U_{\|}^{\mathrm{R}}$ is the final result of the renormalization. The result for renormalized parameters can be represented in a simple form as follows:

$$
\begin{aligned}
& U_{\|}^{\mathrm{R}}=U_{0} \cot \varphi, U_{\perp}^{\mathrm{R}}=U_{0} / \sin \varphi ; \\
& \varphi=U_{0} \ln \left(\frac{T}{T_{\mathrm{GK}}}\right), T_{\mathrm{GK}}=W \exp \left[-\frac{1}{U_{0}} \operatorname{arccot} \frac{U_{\|}}{U_{0}}\right] .
\end{aligned}
$$

The parameter $T_{\mathrm{GK}}$ is a scaling invariant which characterizes properties of the system at low temperatures. Its index GK reminds us that the ground electronic state of the $\mathrm{Yb}^{3+}$ ions is a Kramers doublet. In the case of $J_{\perp}=J_{\|}$, this parameter coincides with the Kondo temperature (Equation (14)). The renormalized Hamiltonian of the exchange interaction between impurities and conduction electrons is obtained simply by substituting in Equation (12) of the initial exchange integrals by the renormalized ones: $J_{\perp} \rightarrow J_{\perp}^{\mathrm{R}}$ and $J_{\|} \rightarrow J_{\perp}^{\mathrm{R}}$.

Due to the extremely small $g_{\|}$-factor, most of the EPR experiments on $\mathrm{YbRh}_{2} \mathrm{Si}_{2}$ were performed in a geometry where both the static and alternating magnetic fields were perpendicular to the tetragonal axis of the crystal. In this case, the renormalized Korringa and Overhauser relaxation rates are the following:

$$
\Gamma_{S S}^{\mathrm{R}}=\frac{\pi}{\hbar} U_{0}^{2} k_{B} T\left(\cot ^{2} \varphi+\frac{3}{4}\right), \Gamma_{\sigma \sigma}^{\mathrm{R}}=\Gamma_{S S}^{\mathrm{R}} \frac{g_{\perp}}{2 g_{\sigma} \rho_{F} k_{B} T} .
$$

Here, we neglected the molecular field appearing due to the RKKY interactions. One can see that both the relaxation rates are logarithmically divergent at temperatures approaching $T_{\mathrm{GK}}$ from above as follows: $\Gamma_{S S}^{\mathrm{R}}, \Gamma_{\sigma \sigma}^{\mathrm{R}} \propto 1 / \ln ^{2}\left(T / T_{\mathrm{GK}}\right)$. Nevertheless, such a behavior does not confirm the belief mentioned above that an observation of the EPR signal is impossible below the Kondo temperature because of its huge line width. This behavior just points out the strong coupling between the spin systems of ions and conduction electrons, and we cannot consider them separately.

The renormalized coupling coefficients for their equations of motion were found to be as follows:

$$
\Gamma_{\sigma S}^{\mathrm{R}}=\frac{\pi}{4 \hbar} U_{0}^{2} \frac{k_{B} T}{\sin ^{2}(\varphi / 2)}, \Gamma_{S \sigma}^{\mathrm{R}}=\frac{g_{\perp}}{2 g_{\sigma} \rho_{F} k_{B} T} \Gamma_{\sigma S}^{\mathrm{R}}
$$

These parameters are also divergent at $T \rightarrow T_{\mathrm{GK}}$ in a way similar to Equation (21), $\Gamma_{S_{\sigma}}^{\mathrm{R}}, \Gamma_{\sigma S}^{\mathrm{R}} \propto$ $1 / \ln ^{2}\left(T / T_{\mathrm{GK}}\right)$. The spin relaxation rates of both spin systems to the lattice (thermal bath) $\Gamma_{S L}$ and 
$\Gamma_{\sigma L}$ remain the same. The result for the relaxation rate of the resonant collective mode at $T>T_{\mathrm{GK}}$ is the following:

$$
\begin{aligned}
& \Gamma_{\text {coll }}^{\mathrm{R}}=\Gamma_{S L}+\Gamma_{\sigma L}^{\mathrm{eff}}+\Gamma_{S S}^{\mathrm{eff}} ; \\
& \Gamma_{\sigma L}^{\mathrm{eff}}=\Gamma_{\sigma L} \frac{\Gamma_{S \sigma}^{\mathrm{R}} \Gamma_{\sigma S}^{\mathrm{R}}}{\left(\Gamma_{\sigma \sigma}^{\mathrm{R}}\right)^{2}}, \Gamma_{S S}^{\mathrm{eff}}=\frac{\left(\Gamma_{S S}^{\mathrm{R}}\right)^{2}-\left(\Gamma_{\sigma S}^{\mathrm{R}}\right)^{2}}{\Gamma_{S S}^{\mathrm{R}}} .
\end{aligned}
$$

The most interesting aspect is the behavior of the relaxation rate at temperatures approaching $T_{\mathrm{GK}}$ from above. In this temperature region, we have $U_{0} \ln \left(T / T_{\mathrm{GK}}\right)=\varphi \rightarrow 0$. After substitution of the renormalized relaxation rates (Equations (21) and (22)) into Equation (23), we obtain the following result for the effective parameters:

$$
\Gamma_{\sigma L}^{\mathrm{eff}}(\varphi \ll 1) \approx\left(\frac{2 g_{\sigma}}{g_{\perp}} \rho_{F} k_{B} T\right) \Gamma_{\sigma L}, \Gamma_{S S}^{\mathrm{eff}}(\varphi \ll 1) \approx \frac{\pi}{8 \hbar} U_{0}^{4} k_{B} T \ln ^{2}\left(\frac{T}{T_{\mathrm{GK}}}\right)
$$

It is wonderful that all divergent contributions became mutually canceled even in the case of anisotropic symmetry. Moreover, the renormalized Korringa relaxation rate of the single impurity (Equation (21)) was transformed in the collective mode in such a way that, instead of a divergence, it goes to zero at $T \rightarrow T_{\mathrm{GK}}$ (Equation (24)). Actually, this result explains the existence of the EPR signal at very low temperatures in the presence of the Kondo effect. As a matter of fact the Kondo effect helps to observe EPR in compounds with a high concentration of magnetic impurities and especially in the Kondo lattices, what is contrary to the common belief [28].

For a detailed comparison of the theory with experimental results, it is necessary to take into account additional interactions of the magnetic impurities and the conduction electrons like spin-phonon, magnetic dipole-dipole, and RKKY interactions and also an inhomogeneity of the static external magnetic field. Some results of these investigations can be found in the review [20].

\section{Conclusions}

The described very different phenomena appearing in different solids at very different phase transitions have a common reason-these transitions strengthen the electron bottleneck regime and stimulate the formation of collective spin excitations of localized and conduction electrons. As a result, we have rather paradoxical situations depending on the concentration of magnetic impurities in superconductors and normal metals. The reason the Hebel-Slichter peak appeared in the NMR experiments at the transition into the superconducting state turned out to be also the reason for its absence in the EPR experiments in superconductors with impurities in the case of their high enough concentrations. The same paradox happens in the case of the Kondo effect, allowing us to observe the EPR signal of the localized electrons below the Kondo temperature instead of it being blocked.

The EPR study of superconducting cuprates was more difficult because of unusually strong spin-phonon interactions of the $\mathrm{Cu}^{2+}$ ions and rather complicated results of the doping process. Nevertheless, Alex Müller was very interested in the EPR investigations of their HTSC properties. To continue this work, he invited an experienced experimenter, A. Shengelaya, to Zürich; I was also later invited for a theoretical support of these investigations. Some of the results are described in the review [29].

Concerning the possibilities of the EPR method, I cannot resist the temptation to quote the concluding words (published already in [29]) of Alex Müller's plenary lecture at the International Conference held in Kazan, dedicated to the 60th year of the EPR discovery:

"60 years after the discovery of EPR in Kazan the method is able to contribute at the forefront in condensed matter physics, such as high temperature superconductivity. This is especially so if properly employed, and the results theoretically interpreted in a scholar way, as well as relate them to other important experiments. Because the EPR spectrometers used are standard, and low cost as are the samples, the research budgets are low; this puts the scientist in a serene mood without stress." 
It seems that the revealed two phenomena described in this communication also confirm these words and one can expect a similar role of the EPR method in future investigations.

Funding: This work received no external funding.

Acknowledgments: I am pleased to acknowledge Y.N. Proshin for reading the manuscript, offering useful comments, and helping to improve it.

Conflicts of Interest: The author declares no conflict of interest.

\section{References}

1. Müller, K.A. Paramagnetic resonance of $\mathrm{Fe}^{3+}$ in single crystals of $\mathrm{SrTiO}_{3}$. Helv. Phys. Acta 1958, 31, 173-204. (In German)

2. Müller, K.A.; Kool, T.W. (Eds.). Properties of Perovskites and Other Oxides; World Scientific Publishing Co. Pte. Ltd.: Singapore, 2010.

3. Barnes, S.E. Theory of electron spin resonance of magnetic ions in metals. Adv. Phys. 1981, 30, 801-938. [CrossRef]

4. Kochelaev, B.I.; Safina, A.M. Electron-Bottleneck Mode for Magnetic Impurities in Metal in the Case of Anisotropic Exchange Interactions. Phys. Solid State 2004, 46, 226-230. [CrossRef]

5. Schrieffer, J.R. Theory of Superconductivity; W.A. Benjamin: Menlo Park, CA, USA, 1964.

6. Hebel, L.C.; Slichter, C.P. Nuclear Spin Relaxation Rate in Normal and Superconducting Aluminium. Phys. Rev. 1959, 113, 1504-1519. [CrossRef]

7. Redfield, A.G. Nuclear Spin Relaxation Time in Superconducting Aluminum. Phys. Rev. Lett. 1959, 3, 85-86. [CrossRef]

8. Hebel, L.C. Theory of Nuclear Spin Relaxation in Superconductors. Phys. Rev. 1959, 116, 79-81. [CrossRef]

9. Rettori, R.; Davidov, D.; Chaikin, P.; Orbach, R. Magnetic Resonance of a Localized Magnetic Moment in the Superconducting State: LaRu2:Gd. Phys. Rev. Lett. 1973, 30, 437-440. [CrossRef]

10. Davidov, D.; Rettori, R.; Kim, H.M. Electron-spin resonance of a localized moment in the superconducting state: BRu2:Gd (B=La,Ce,Th). Phys. Rev. B 1974, 9, 147-153. [CrossRef]

11. Tagirov, L.R.; Trutnev, K.F. Spin kinetics and EPR in superconductors. J. Phys. F Met. Phys. 1987, 17, 695-713. [CrossRef]

12. Alekseevsky, N.E.; Garifullin, I.A.; Kochelaev, B.I.; Kharakhashyan, E.G. Electron resonance with localized magnetic moments of Er in superconducting La. Sov. Phys. JETP Lett. 1973, 18, 189-191.

13. Alekseevsky, N.E.; Garifullin, I.A.; Kochelaev, B.I.; Kharakhashyan, E.G. Electron paramagnetic resonance for localized magnetic states in the superconducting La-Er system. Sov. Phys. JETP 1977, 45, 799-804.

14. Kochelaev, B.I.; Kharakhashyan, E.G.; Garifullin, I.A.; Alekseevsky, N.E. Electron paramagnetic resonance of localized moments in a type-II superconductor. In Proceedings of the 18th AMPERE Congress, Nottingham, UK, 9-14 September 1974; North-Holland Pub. Co.: Amsterdam, The Netherlands, 1975; pp. 23-24.

15. Maki, K. Theory of Electron-Spin Resonance in Gapless Superconductors. Phys. Rev. B 1973, 8, 191-199. [CrossRef]

16. Kosov, A.A.; Kochelaev, B.I. Electron paramagnetic resonance on the localized magnetic moments in superconductors. Sov. Phys. JETP 1978, 47, 75-83.

17. Kochelaev, B.I.; Tagirov, L.R.; Khusainov, M.G. Spatial dispersion of the spin susceptibility of conduction electrons in superconductors. Sov. Phys. JETP 1979, 49, 291-301.

18. Kochelaev, B.I.; Tagirov, L.R. “Exchange-Field-Narrowing” process for the inhomogeneously broadened EPR lines in superconductors. Solid State Commun. 1985, 53, 961-966. [CrossRef]

19. Bogolyubov, N.N.; Tyablikov, S.V. On an application of perturbation theory to the polar model of a metal. Zhurnal Ehksperimental'noj i Teoreticheskoj Fiziki 1949, 19, 251-255.

20. Kochelaev, B.I. Magnetic properties and spin kinetics of a heavy-fermion Kondo lattice. Low Temp. Phys. 2017, 43, 93-103. [CrossRef]

21. Kondo, J. Resistance Minimum in Dilute Magnetic Alloys. Progr. Theor. Phys. 1964, 32, 37-49. [CrossRef]

22. Baberschke, K.; Tsang, E. ESR Study of the Kondo Effect in Au:Yb. Phys. Rev. Lett. 1980, 45, 1512. [CrossRef]

23. Abrikosov, A.A. Electron scattering on magnetic impurities in metals and anomalous resistivity effects. Physics 1965, 2, 5-20. [CrossRef] 
24. Suhl, H. Dispersion Theory of the Kondo Effect. Phys. Rev. 1965, 138, 515-522. [CrossRef]

25. Sichelschmidt, J.; Ivanshin, V.A.; Ferstl, J.; Geibel, C.; Steglich, F. Low Temperature Electron Spin Resonance of the Kondo Ion in a Heavy Fermion Metal: YbRh2Si2. Phys. Rev. Lett. 2003, 91, 156401. [CrossRef]

26. Sichelschmidt, J.; Wykhoff, J.; Krug von Nidda, H.-A.; Ferstl, J.; Geibel, C.; Steglich, F. Spin dynamics of $\mathrm{YbRh}_{2} \mathrm{Si}_{2}$ observed by electron spin resonance. J. Phys. Condens. Matter 2007, 19, 116204. [CrossRef]

27. Anderson, P.W. A poor man's derivation of scaling laws for the Kondo problem. J. Phys. C Solid State Phys. 1970, 3, 2436-2441. [CrossRef]

28. Kochelaev, B.I.; Belov, S.I.; Skvortsova, A.M.; Kutuzov, A.S.; Sichelschmidt, J.; Wykhoff, J.; Geibel, C.; Steglich, F. Why could electron spin resonance be observed in a heavy fermion Kondo lattice? Eur. Phys. J. B 2009, 72, 485-489. [CrossRef]

29. Kochelaev, B.I. Electron Paramagnetic Resonance in Superconducting Cuprates. In Springer Series in Materials Science 255: High-Tc Copper Oxide Superconductors and Related Novel Materials Dedicated to Prof. K. A. Müller on the Occasion of His 90th Birthday; Bussmann-Holder, A., Keller, H., Bianconi, A., Eds.; Springer: New York, NY, USA, 2017; pp. 165-175.

(C) 2020 by the author. Licensee MDPI, Basel, Switzerland. This article is an open access article distributed under the terms and conditions of the Creative Commons Attribution (CC BY) license (http://creativecommons.org/licenses/by/4.0/). 